\title{
On the massive star contents of Cygnus OB2^
}

\author{
F. Comerón ${ }^{1}$, A. Pasquali ${ }^{2}$, G. Rodighiero ${ }^{3}$, V. Stanishev $^{4}$, E. De Filippis ${ }^{5}$, B. López Martí ${ }^{6}$, \\ M. C. Gálvez Ortiz ${ }^{7}$, A. Stankov ${ }^{8}$, and R. Gredel ${ }^{9}$
}

1 European Southern Observatory, Karl-Schwarzschild-Str.2, 85748 Garching bei München, Germany

2 Space Telescope European Coordinating Facility, Karl-Schwarzschild-Str.2, 85748 Garching bei München, Germany

e-mail: apasqual@eso.org

3 Dipartimento di Astronomia, Università di Padova, vicolo dell'Osservatorio 2, 35122 Padova, Italy e-mail: rodighiero@pd.astro.it

4 Institute of Astronomy, Bulgarian Academy of Sciences, 72 Tsarigradsko Shousse, Blvd, 1784 Sofia, Bulgaria e-mail: vall@astro.bas.bg

5 Astrophysics Research Institute, Liverpool John Moores University, Twelve Quays House, Egerton Wharf, Liverpool CH41 1LD, UK

e-mail: bdf@astro.livjm.ac.uk

6 Thüringer Landessternwarte, Sternwarte 5, 07778 Tautenburg, Germany

e-mail: belen@tls-tautenburg.de

7 Facultad de Ciencias Físicas, Universidad Complutense de Madrid, 28040 Madrid, Spain e-mail: mcz@astrax.fis.ucm.es

8 ESTEC, Research \& Scientific Support Department (SCI-SR), Keplerlaan 1, 2201 AZ Noordwijk, The Netherlands e-mail: astankov@rssd.esa.int

9 Max-Planck Institut für Astronomie, Königstuhl 17, 69117 Heidelberg, Germany

e-mail: gredel@caha.es

Received 18 July 2001 / Accepted 25 April 2002

\begin{abstract}
We present a near-infrared spectroscopic survey of a large area centered on the Cygnus OB2 association aimed at constraining its massive star contents. Our goal is to establish a nearly complete list of O-type members of the association, both to examine recent claims based on starcounts that suggest a richer content than previously thought, and to provide a suitable database for further studies of the entire high-mass end of one of the richest associations of the Galaxy. The target selection is based on the $J H K$ photometry published in the 2MASS all-sky survey. We identify 46 new early-type candidates, most of them expected to be O-type stars, plus 16 new stars with emission in $\operatorname{Br} \gamma$ and often in other lines as well, characteristic of evolved massive stars undergoing intense mass loss. We also present spectra of three luminous stars with CO overtone emission, one of them having also intense $\mathrm{H}_{2}$ emission and being associated with compact nebulosity. By considering our findings, those of other authors, and plausible completeness corrections, we estimate the number of O-type stars or stars having evolved from a O-type progenitor to be 90-100, slightly below, but compatible with, most recent starcounts estimates by Knödlseder (2000, A\&A, 360, 539). These results support the notion that Cygnus OB2 may be considered as a young globular cluster. The lists of new members that we provide, in particular those with emission lines, should be a useful resource for future investigations of Cygnus OB2 itself, as well as of very massive stellar evolution by providing a nearby, abundant sample of stars sharing a common environment.
\end{abstract}

Key words. stars: early type - stars: luminosity function, mass function - open clusters and associations: individual: Cygnus OB2

\section{Introduction}

The association Cygnus OB2 (Johnson \& Morgan 1954; Schulte 1958; Reddish et al. 1966) is one of the richest

\footnotetext{
Send offprint requests to: F. Comerón,

e-mail: fcomeron@eso.org

* Based on observations collected at the German-Spanish Astronomical Center, Calar Alto.
}

aggregates of massive stars in our Galaxy, and includes some of the most luminous stars known. The large extinction in its direction renders it rather inconspicuous despite its relative proximity to the Sun $(1.7 \mathrm{kpc}$; Torres-Dodgen et al. 1991), and is the main reason why a complete census of even its most massive members is as yet unavailable. The combination of a well sampled upper end of the stellar mass function, proximity, and presence of objects 
displaying the singular phenomena that manifest themselves only at extremely high masses, makes Cygnus OB2 a very attractive laboratory for the study of both the individual and collective properties of the most massive stars. It also stresses the need for a systematic observational effort to precisely determine its stellar contents in order to provide a firm database for such studies. Indeed, wide-field $U B V$ CCD imaging (Massey \& Thompson 1991; Massey et al. 1995) and objective prism surveys in the red (Parthasarathy \& Jain 1995) strongly suggest an abundant contents of highly reddened OB stars, but detailed spectral classification and even definite membership determination is still missing for most of the objects revealed by those and other works. The potential reward for such studies is hinted at by the more recent work of Knödlseder (2000) who, on the basis of a statistical study of point sources revealed by the 2MASS near-infrared survey, suggested an even richer stellar contents and extent of Cygnus OB2, and proposed its reclassification as a young globular cluster rather than a normal OB association.

In this paper we present the results of a near-infrared spectroscopy survey of a large field covering most of the area occupied by the association. Our targets were selected on the basis of their position in the near-infrared color-magnitude diagram as potential early-type members. Most of them are not included in previous surveys at visible wavelengths because of substantial extinction in their direction. Our results show a variety of spectra corresponding to early-type stars that are likely members of the association, and to late-type background stars. Among the former, a number of them display emission in the Brackett $\gamma$ line, characteristic of the high mass loss rates typical of evolved stages, and a few have the $\mathrm{H}_{2}$ and $\mathrm{CO}$ emission indicative of abundant circumstellar molecular material. The numbers of O-type stars that we derive, with most of these stars having available visible or near-infrared spectra supporting their classification as such, confirm recent estimates that suggest the reclassification of Cygnus OB2 as a young globular cluster.

\section{Observations}

The majority of the observations presented in this paper were obtained in an observing run at the $1.23 \mathrm{~m}$ telescope in Calar Alto, using the near-infrared camera MAGIC, between 25 August and 7 September 2001. A first, small subset of observations was also obtained during a summer school held at Calar Alto in July 2000 (http://www.iap.fr/eas/neon.html), using identical instrumentation and setup. We used a grism covering both the $K$ and most of the $H$ bands in a single setup, in combination with a slit yielding a width of 2 pixels on the detector. This setup allowed us to obtain spectra covering the $1.50-2.40 \mu \mathrm{m}$ range at a resolution of $R \simeq 240$. Besides the spectra of the Cygnus OB2 targets we also obtained spectra of the G5IV star HD 190771 at approximately one-hour intervals. This star, assumed to be essentially featureless at the resolution employed, lies near Cygnus OB2 in the sky and was used to monitor telluric absorption. For each source we obtained six spectra at positions evenly spaced along the slit, each spectrum being a stack of individual $6 \mathrm{~s}$ exposures. The number of individual exposures at each position varied between 10 and 20, depending on the cataloged $K$ magnitude of the target. Each frame was dark-subtracted and flat-fielded, and individual spectra were extracted and wavelengthcalibrated using $\mathrm{OH}$ airglow lines (Oliva \& Origlia 1992). The individual, wavelength-calibrated spectra were then combined separately for each program object and for the telluric reference star, and the spectrum of each program star was ratioed by that of the telluric reference star obtained closest in time. To achieve relative flux calibration the resulting spectrum was finally multiplied by a blackbody of $5700 \mathrm{~K}$ temperature, which should adequately represent the spectral energy distribution of the telluric reference star in the near infrared.

A few targets of special interest because of their spectra or their association with sources at longer wavelengths were imaged through the broad-band $J H K_{M}$ filters with the same telescope and instrument. In imaging mode, the detector yields a field of view of $4^{\prime} 9 \times 4^{\prime} 9$ at a scale of $1^{\prime \prime} 15$ per pixel. Individual images of the field around each of these stars were obtained using a dither pattern of 9 points on a $3 \times 3$ points grid, adding up 12 integrations of $5 \mathrm{~s}$ each at every grid position. A sky frame was constructed for each field and filter by median-filtering its component frames uncorrected for the motions of the telescope between consecutive grid points, so as to remove stellar images. The sky frame was subtracted from each image in the dither, and the resulting dark-subtracted, flat-fielded, and sky-subtracted images were then aligned and coadded.

\section{Target selection}

We used the Second Incremental Release of the 2MASS Point Source Catalog to extract a sample of stars within one degree of the center of Cygnus OB2, assumed to lie near the position of the multiple, Trapezium-like system VI Cyg 8 (Schulte 1958). This area contains practically the full extent of the association as determined by Knödlseder (2000). Figure 1 shows the $(H-K), K$ diagram of the region together with the limits corresponding to stars earlier than a given spectral type having different amounts of foreground reddening. Cygnus OB2 members earlier than spectral type $S$ are expected to fulfill

$K<\left[M_{K}(S)-1.78(H-K)_{0}(S)+11.2\right]+1.78(H-K)(1)$

where $M_{K}$ is the absolute $K$ magnitude, $(H-K)_{0}$ is the intrinsic color (which we take from the compilations by Drilling \& Landolt 2000 and Tokunaga 2000), 11.2 is the adopted distance modulus to Cygnus OB2, and the 1.78 factor arises from the slope of the reddening vector in the $(H-K), K$ diagram using the Rieke \& Lebofsky (1985) extinction law. This condition is obviously fulfilled 


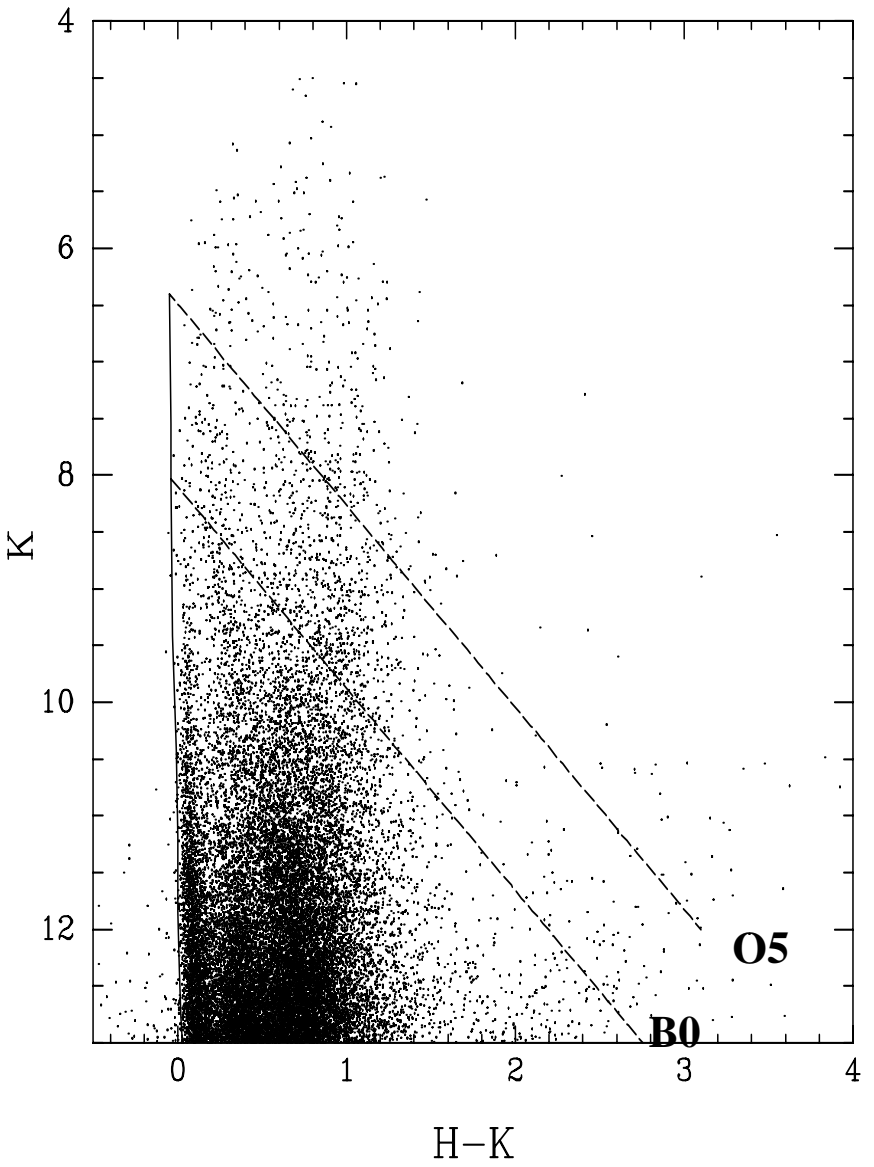

Fig. 1. $(H-K), K$ diagram of all the 2MASS sources detected within 1 degree of the adopted center of Cygnus OB2. The nearly vertical line on the left is the position of the unreddened main sequence at the distance of $1.7 \mathrm{kpc}$, and the dashed lines indicate the locus expected for stars of different spectral types at that same distance reddened by different amounts.

also by bright, background late-type stars, unrelated to the $\mathrm{OB}$ association.

Figure 2 shows the $(J-H),(H-K)$ diagram of the stars brighter than the limit given by Eq. (1) when using the values of $M_{K}(S)$ and $(H-K)_{0}(S)$ appropriate for a spectral type B0, showing the signature of the presence of the association. The extinction vector traced by the distribution of heavily reddened stars is well represented by the extinction law of Rieke \& Lebofsky (1985). This was already shown to be so by Torres-Dodgen et al. (1991) in the infrared, who also confirmed early findings by Johnson \& Borgman (1963) at visible wavelengths. At lowto-moderate values of the color indices there are clearly two parallel sequences each of which follows the reddening vector. The upper one has its origin at the position occupied by unreddened late-type giants, while the lower one starts at the position of the earliest stars. This alone already may be used to obtain a first estimate of the earlytype contents of the association and of the average reddening in its direction, by just tracing back the position of the points in the lower sequence along the reddening vector until intersecting the locus of unreddened early-type

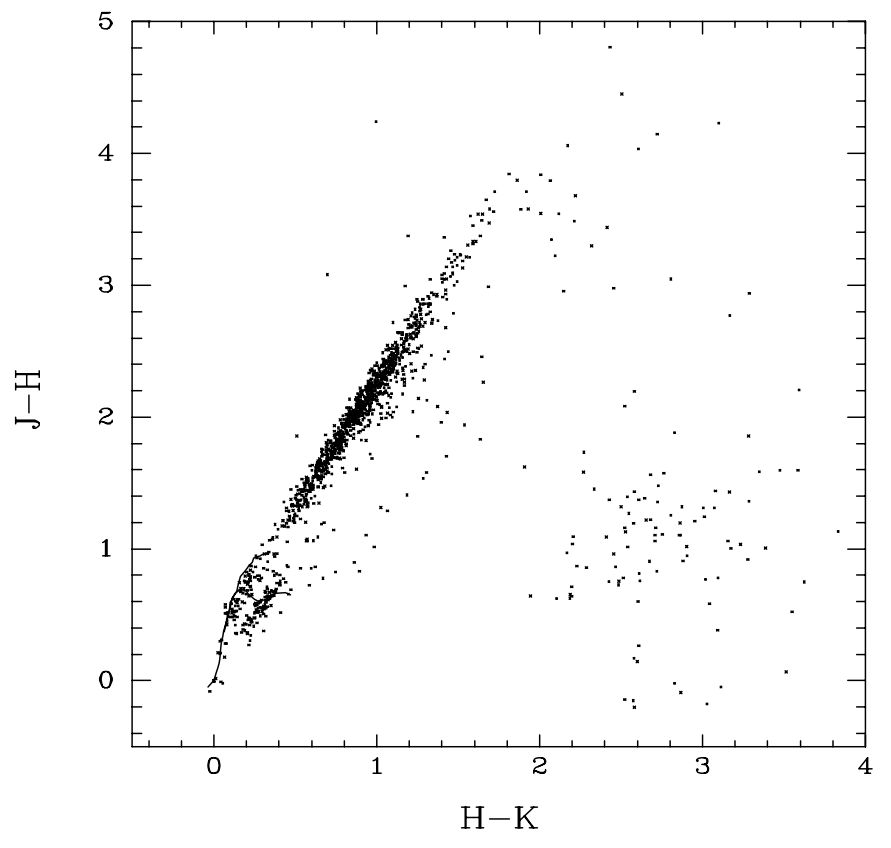

Fig. 2. $(H-K),(J-H)$ diagram of all the 2MASS sources detected within 1 degree of the adopted center of Cygnus OB2 and above the limiting line for spectral type B0 in Fig. 1. The solid lines at the lower left of the figures mark the expected positions for unreddened main sequence (lower branch) and giant (upper branch) stars.

stars. Another obvious group of objects in Fig. 2 appears at the highest values of $(H-K)$, while having relatively blue $(J-H)$, probably indicative of the existence of strong infrared excesses of circumstellar origin. Nearly all these objects are relatively faint $(K>10)$ and, although they are of great interest to study the ongoing star forming activity in Cygnus OB2, their faintness in the bands that are less affected by circumstellar emission suggests that they may be intermediate mass stars, rather than members of the high end of the mass function of Cygnus OB2 that is the main purpose of our study. Their faintness also places them outside the limits accessible within moderate integration times with the telescope and instrument used for this survey, and therefore we will not discuss them in the present paper. The reader is referred to comprehensive studies on IRAS sources or molecular gas in the Cygnus region (Odenwald 1989; Odenwald \& Schwartz 1989; Dobashi et al. 1996) for discussions on the youngest component of this region.

Most of the objects near the lower sequence in Fig. 2 have relatively blue colors, indicating that much of the early-type stellar contents of Cygnus OB2 is only moderately reddened. The upper sequence dominates at redder colors, showing that the vast majority of red stars seen in the direction of Cygnus OB2 are actually background, unrelated late-type stars. However, there is still a considerable number of stars in the region lying to the right of the upper sequence in the color-color diagram. These infrared-bright, very red objects are in principle candidate members of the heavily reddened massive component of 
Cygnus OB2 whose existence has been suggested by previous works (Reddish et al. 1966; Partharasathy \& Jain 1995; Knödlseder 2000). Contrarily to what happens with the lightly obscured component, these objects do not tend to align along the extension of the lower sequence. This may be due to factors such as peculiarities in their intrinsic colors, circumstellar emission by hot dust, anomalous behavior of the extinction law in their direction, or contamination by late-type stars like long-period Mira variables, whose intrinsic $H-K$ is redder than that of normal giants with the same intrinsic $J-H$ (Glass et al. 1995). The spectroscopy that we present in this paper should help in clarifying the nature of these objects.

We made a first selection of targets for spectroscopic observations by using Eq. (1) in combination with the estimated sensitivity limit of $K<10$ of the telescope and instrument used within a reasonable integration time. We adopted a limiting spectral type B0 in Eq. (1), corresponding to $M_{K}=-3.1,(H-K)_{0}=-0.04$. The $K<10$ condition implies some incompleteness affecting possible highly obscured members of the association, appearing at $(H-K)=1.1$ (corresponding to $\left.A_{V}>17.5\right)$ for B0 stars, and even higher extinctions for earlier types. However, as we discuss below in view of the location of the actually observed stars in the $(J-H),(H-K)$ diagrams, we do not expect such incompleteness to affect our results in a significant way.

The selection criteria given by Eq. (1) and $K<10$ reduced the sample of 2MASS stars within one degree from the center of Cygnus OB2 to 985. We further constrained our sample by calculating for each star its distance in the $(J-H),(H-K)$ diagram to the upper reddening vector, and retaining only those with a distance greater than $\sim 0.1$ mag. As can be seen from Fig. 2 this is a rather generous cutoff that actually includes a large fraction of stars closer to the upper than to the lower sequence. In this way we produced a list of 397 star, that we further divided into two priority groups separated by $(H-K)$ greater or smaller than 0.5 . We gave higher priority to the more reddened group (corresponding to $A_{V}>8$ approximately), since it is here where we would expect to find the largest fraction of previously unidentified Cygnus OB2 members and the bulk of the hypothesized obscured population. We observed the 232 stars in this group. The available observing time allowed us to obtain spectra also for 83 stars in the second, bluer group, namely about half of our initially planned targets in this group. We proceeded by order of decreasing distance to the upper reddening sequence reaching a minimum distance of $\sim 0.2$ mag to it (as compared to $\sim 0.1$ for the redder sample), thus observing nearly all the objects clustering around the lower reddening sequence. To assess the possible incompleteness of our spectroscopically observed sample with respect to the total population of O-type members of Cygnus OB2 induced by our $K<10$ cutoff, Figs. 3 and 4 plot the colormagnitude and color-color diagrams like in Figs. 1 and 2 respectively, but now highlighting the objects for which we obtained spectra. These figures suggest that indeed we

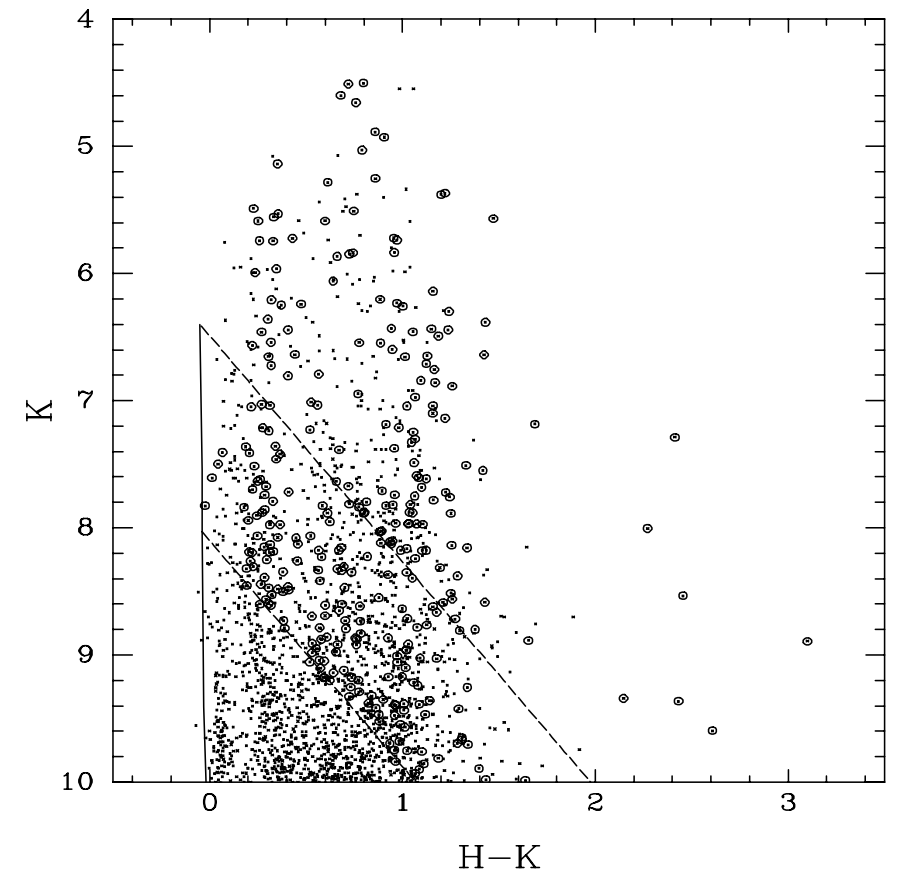

Fig. 3. Same as Fig. 1, but now marking with a small circle the stars for which spectra have been obtained. Most of the non-observed stars redder than $H-K=0.5$ and brighter than $K=10$ lie very close to the reddening vector that has its origin at the locus of the late-type giants, and therefore are not expected to be members of Cygnus OB2. Some other stars in this region of the color-magnitude diagram are in the region of strong infrared excess (lower right part) of Fig. 2 and are therefore unlikely to be members of Cygnus OB2 as well for the reasons discussed in the text. Most of the stars with $H-K<0.5$ and $J H K$ colors suggestive of an early-type were also observed spectroscopically.

should expect to have sampled the vast majority of the O-star contents of the association, especially the more obscured members.

\section{Results}

While the spectra obtained in this survey lack the resolution and signal-to-noise ratio needed to produce an accurate spectral classification of each target, they are nevertheless useful for assigning them to one of several broad categories, as well as to guide future detailed investigations of particular objects.

Both the existence of lightly obscured members of Cygnus OB2 and the absence of any significant nearby intervening cloud over the surveyed area, which would stand out as a decrease in stellar density in visible-light images of the region, suggest that most of the extinction on the objects plotted in the 2MASS color-color and color-magnitude diagrams is produced at a distance similar to that of Cygnus OB2 or greater. Our selection of targets that are both very red and bright in the infrared thus ensures that they are intrinsically bright, thus implying that the vast majority should fall within one of two categories: hot luminous young stars likely to belong to 


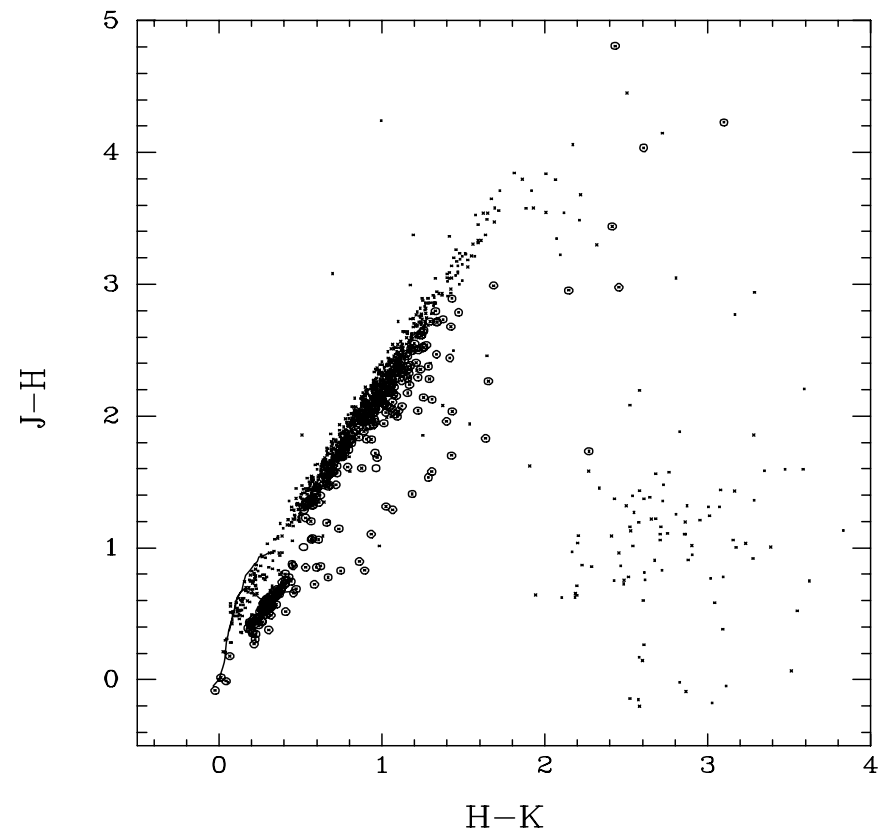

Fig. 4. Same as Fig. 2, but now marking with a small circle the stars for which spectra have been obtained. This figure shows that our spectroscopically observed sample with $H-K>0.5$ extends well into the band dominated by reddened late-type giants, thus providing a highly complete sampling of the possible obscured early-type component of Cygnus OB2. No stars from the region of high infrared excess were spectroscopically observed due to their faintness; as discussed in the text, these stars are unlikely to be a part of the massive component of the association. This might not be the case for at least some of the relatively few stars near the upper end of main reddened band, which are also too faint $(K>10)$ to be included in our sample.

the association, and cool red giants and supergiants. The distinction between both groups can be established easily from our spectra, mainly based on the presence or absence of $\mathrm{CO}$ bands in absorption longwards of $2.29 \mu \mathrm{m}$. Even for early K-type giants with less prominent $\mathrm{CO}$ bands that may not be clearly seen in some of our noisier spectra, the shape of the continuum still allows a clear distinction between them and the much hotter early-type stars whose infrared spectral energy distribution is well matched by a reddened Rayleigh-Jeans tail. Of the cool stars group, only red supergiants might belong to the association on the basis of their ages and evolutionary status, a possibility that we will consider below.

\subsection{Early-type stars}

At the resolution and signal-to-noise of the spectra under consideration here $\mathrm{O}$ - and early B-type stars with a purely absorption-line spectrum should look featureless, the noise dominating over the depth of the strongest features due to $\mathrm{H}$ and He. Lacking more information from the current material, we thus classify as candidate early-type stars in our sample those that appear as a featureless continuum.

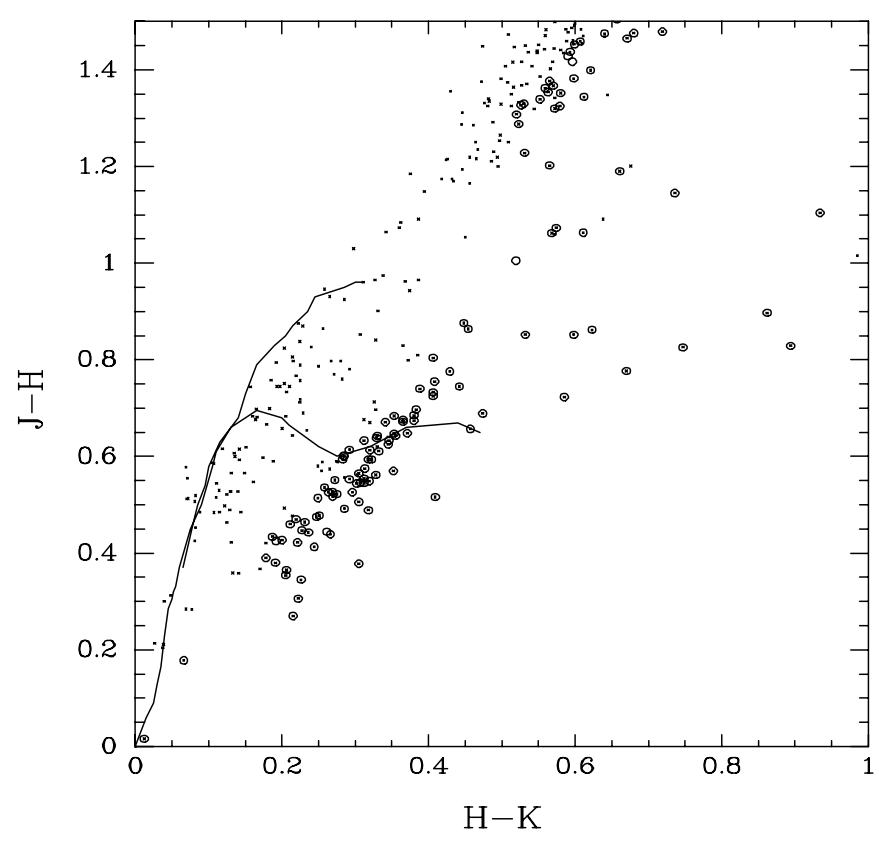

Fig. 5. A detail of Fig. 4 focusing on the region with $H-K<$ 0.5. Although our observations clearly include the majority of stars with colors consistent with them being early-type, lightlyreddened members of Cygnus OB2, the completeness of the spectroscopic sample in this color range is expected to be less than that in the range $H-K>0.5$. This incompleteness is partly overcome by the adoption of spectroscopic classifications in the visible that are available in the literature for many of these stars. In any case, the obvious gap seen at $H-K<0.5$ between the group of stars spectroscopically observed and the non-observed ones lying above them (many of which may be foreground stars) argues against a significant incompleteness of our spectroscopic sample over the full color range.

We have thus identified 77 early-type candidates in our sample. Of these, 68 belong to the "blue" group $(H-K)<0.5$, and many of them have therefore been included in surveys in the visible. Accurate spectral classifications are available in the literature for 31 of them, mostly from the works of Massey \& Thompson (1991) and Hanson et al. (1996). Most of these stars are O-type (24). The remaining 7 have been classified in the literature as B0V (2), B1.5V (1), B1II (1), or simply B (3). Detailed studies on some of the most luminous among these objects can be found in Herrero et al. (1999). Confirmed contamination among early-type candidates by non-OB stars in the blue group is found to be small, as expected from the small distance of the sample to the reddening band of early-type objects in the infrared color-color diagram: one star in this group has also a published classification as F5 and is thus foreground, and four other stars that were included in the blue sample on the basis of their infrared colors turned out to display clearly visible hydrogen absorption lines classifying them as foreground A-type stars, confirmed in three cases by published spectral classifications in the visible.

The remaining 37 stars having featureless spectra in the group of $(H-K)<0.5$, as well as the 9 stars with 


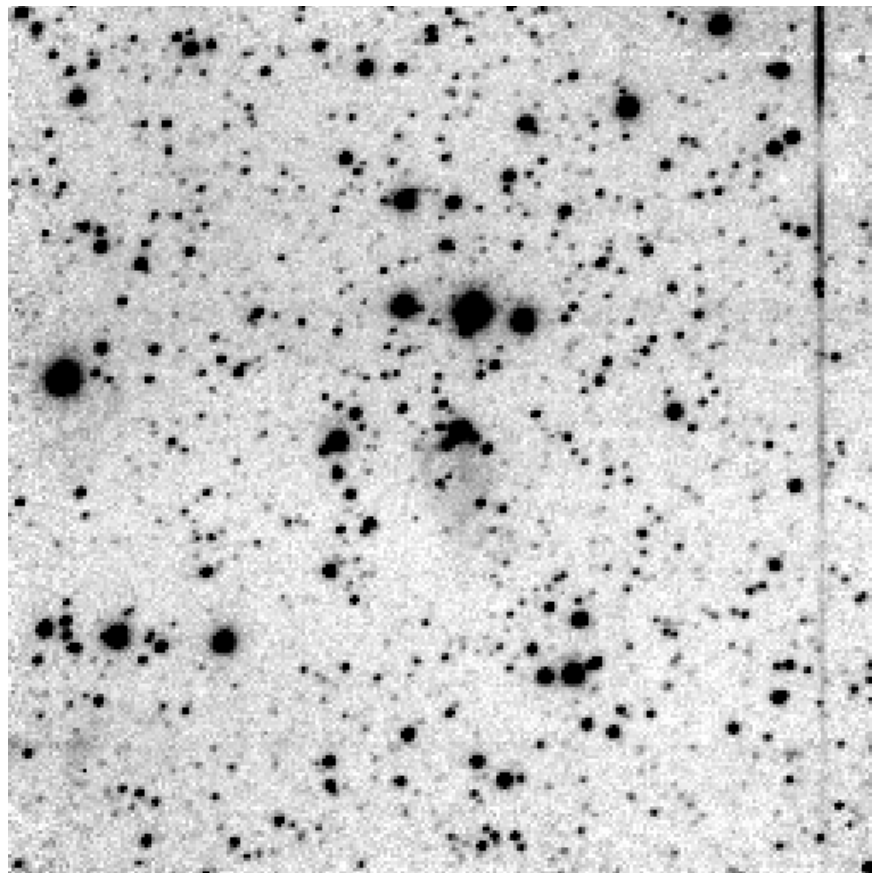

Fig. 6. $K$-band image of star $\mathrm{A} 1$, showing the existence of a faint compact nebulosity around it. The field of view is $5^{\prime} \times 5^{\prime}$, with North at the top and East to the left.

featureless spectra in the $(H-K)>0.5$ group, are new candidate members of the association and are listed in Table 1. None of the latter appears to be associated to sources seen at other wavelengths, with the exception of A1 whose position is consistent with that of the radiocontinuum source MITG J2033+4041 (Griffith et al. 1991) appearing in the near-infrared as a small, faint HII region around this star (Fig. 6). Given the limited positional accuracy of the MITG catalog $\left(\sim 40^{\prime \prime} \mathrm{rms}\right)$, the $K$-band image confirms the association of the star with the HII region. Some of the stars in Table 1 had been already included in the lists of Reddish et al. (1966), Massey \& Thompson (1991) and Parthasarathy \& Jain (1995), but spectral classification was not available. At least two of these new members seem to be the counterparts of stellar soft X-ray sources detected by the Einstein satellite, a property that is common among the brigther members of Cygnus OB2 (Waldron et al. 1998).

\subsection{Stars with Bry in emission}

Our survey also revealed 20 stars with discernible emission in $\operatorname{Br} \gamma(2.166 \mu \mathrm{m})$ and occasionally in other lines as well. Besides these 20 stars, our selection criteria also included the three Wolf-Rayet stars WR 144, WR 146, and V1923 Cyg. The complete list is given in Table 2, and the full set of spectra appears in Fig. 7. The equivalent widths of a number of features is listed in Table 3. Only 4 of these emission-line stars have published spectra in the literature: these are Cyg OB2 \# 360 (B1.5III...; Massey \& Thompson 1991), VI Cyg 9 (O5Iab:e; Herrero et al. 1999), VI Cyg 11 (O6e; Herrero et al. 1999), and Cyg OB2 \# 708
(B1.5V and Herbig Ae/Be; Thé et al. 1994). Three other stars with $\mathrm{Br} \gamma$ emission have been included in previous lists of Cygnus OB2 members but without published spectral types, including V2188 Cyg (Pigulski \& Kolaczkowski 1998). We also note that three of the reddest objects are probably associated with IRAS point sources.

Despite the common feature of having $\operatorname{Br} \gamma$ in emission, Fig. 7 shows a variety of spectral morphologies found among these objects. The features in their spectra are characteristic of those displayed in late stages of massive star evolution (WNL, Of, Ofpe/WN9, Be, B[e], and LBVs), but assigning the different classes of emission-line spectra that we obtain to any of those stages is not possible. In their extensive review on the near-infrared properties of massive stars in transition, Morris et al. (1996) stress the difficulty of assigning unambiguous spectroscopic classification criteria to separate among the different groups established from observations in the visible, finding evidence for a close relationship among them. A study of our objects to a comparable level of detail requires better quality spectra than currently available and is clearly beyond the scope of this paper, but the results plotted in Fig. 7 already provide a glimpse at the diversity found also in our sample. For example, it is interesting to note the differences in the Brackett decrement between the group formed by B9, B10, B11, B12, B13, B15, and B16 on one side, and B14 and B18 (at least; the $H$-band spectra of B3, B7, and B8 are too faint for a reliable measurement of the higher Brackett lines) on the other. The systematic difference is reminiscent of that found by Morris et al. between Ofpe/WN9 stars and LBVs. The HeI line at $2.058 \mu \mathrm{m}$ is clearly seen in B10 and B14, and is possibly present also in B9, B12, B13, and B15, while the HeI line at $1.700 \mu \mathrm{m}$ is seen in B16 and B17. Wide variations of this line have been measured by Morris et al. within each of the groups of evolved massive stars mentioned above.

$K$-band images obtained for one of our reddest targets, B3, shows a compact, asymmetric nebula roughly $30^{\prime \prime}$ across around it (Fig. 8). Both the mid-infrared point source IRAS $20327+4120$ and the water maser emission detected near that position by Palla et al. (1991) are likely to arise in this nebulosity. We finally note the reddest object in our sample, B1, whose spectrum is featureless with the exception of a clearly discernible emission line at $2.225 \mu \mathrm{m}$. Weak $\operatorname{Br} \gamma$ emission is also probably present. The stronger emission might be due to [FeII], sometimes observed in the spectra of LBVs (see the spectra of WRA 751 in Morris et al. 1996) but not as a dominant or unique emission feature. B1 is probably associated to IRAS $20343+4129$, which has been detected as a faint, unresolved thermal radiosource at $6 \mathrm{~cm}$ by Miralles et al. (1994). Its mid-infrared properties and the association with dense gas traced by $\mathrm{NH}_{3}$ (Miralles et al. 1994) agree with it being an ultracompact HII region. However, the brightness at $K$ argues against B1 and IRAS 20343+4129 being the same object, as the central star of the HII region should be deeply embedded and highly obscured even in the near-infrared. Clearly, the nature of B1 and its 
Table 1. Candidate new early-type members of Cygnus OB2 with featureless low-resolution spectra.

\begin{tabular}{|c|c|c|c|c|c|c|}
\hline Number & $\alpha(2000)$ & $\delta(2000)$ & $K$ & $(J-H)$ & $(H-K)$ & remarks \\
\hline $\mathrm{A} 1$ & 203311.7 & 404154.4 & 9.987 & 1.83 & 1.637 & possibly associated to radio-continuum source [1] \\
\hline $\mathrm{A} 2$ & 203251.4 & 41840.0 & 9.696 & 1.534 & 1.285 & \\
\hline A3 & 203133.8 & 411915.4 & 9.932 & 1.29 & 1.066 & \\
\hline $\mathrm{A} 4$ & 203136.3 & 41223.3 & 5.865 & 1.19 & 0.661 & \\
\hline A5 & 20359.8 & 413529.7 & 7.885 & 1.063 & 0.611 & \\
\hline A6 & 20328.3 & 40257.0 & 9.104 & 1.073 & 0.574 & \\
\hline A7 & 203443 . & 402930.3 & 9.042 & 1.062 & 0.568 & \\
\hline A8 & 203341.6 & 414757.1 & 6.792 & 1.202 & 0.565 & \\
\hline A9 & 203532.7 & 412055.2 & 9.056 & 1.005 & 0.519 & \\
\hline A10 & 203455.1 & 403444.0 & 8.261 & 0.864 & 0.454 & \\
\hline A11 & 203231.5 & $41 \quad 148.0$ & 6.637 & 0.745 & 0.442 & possibly associated to X-ray source $1 \mathrm{E} 203043+4103.9[2]$ \\
\hline A12 & 203338.2 & 40416.0 & 5.723 & 0.776 & 0.429 & \\
\hline A13 & 20331.2 & 403233.0 & 8.491 & 0.755 & 0.408 & \\
\hline A14 & 203118.9 & 42256.0 & 8.464 & 0.725 & 0.406 & \\
\hline A15 & 203136.8 & 40599.0 & 6.805 & 0.733 & 0.406 & \\
\hline A16 & 203436.9 & 40412.0 & 8.789 & 0.74 & 0.388 & \\
\hline A17 & 203235.3 & 411445.0 & 8.731 & 0.697 & 0.383 & \# $886[3]$ \\
\hline A18 & 20307.8 & 412350.0 & 8.347 & 0.685 & 0.38 & \\
\hline A19 & 203125.9 & 41162.0 & 8.504 & 0.674 & 0.38 & $\# 597[4]$ \\
\hline $\mathrm{A} 20$ & 20332.9 & 404725.0 & 6.248 & 0.648 & 0.371 & possibly associated to X-ray source $2 \mathrm{E} 2031.2+4037$ [5] \\
\hline $\mathrm{A} 21$ & 202934.8 & 41208.0 & 8.483 & 0.647 & 0.353 & \\
\hline $\mathrm{A} 22$ & 203311.2 & 404233.0 & 8.076 & 0.684 & 0.353 & \\
\hline $\mathrm{A} 23$ & 203039.7 & 41848.0 & 5.963 & 0.633 & 0.346 & \\
\hline $\mathrm{A} 24$ & 203444.1 & 405158.0 & 7.461 & 0.625 & 0.345 & \\
\hline A 25 & 203238.4 & 404044.0 & 7.358 & 0.671 & 0.341 & \\
\hline A 26 & 203057.7 & 41957.0 & 8.187 & 0.643 & 0.33 & \\
\hline $\mathrm{A} 27$ & 203444.7 & 405146.0 & 5.745 & 0.638 & 0.329 & \\
\hline $\mathrm{A} 28$ & 203416 . & 41219.0 & 8.526 & 0.594 & 0.322 & \\
\hline $\mathrm{A} 29$ & 203456 . & 403818.0 & 6.541 & 0.594 & 0.318 & \\
\hline A30 & 203122.1 & 41123.0 & 8.61 & 0.489 & 0.318 & \\
\hline A31 & 203239.5 & 405247.0 & 7.976 & 0.633 & 0.312 & KMJ 1718 [6] \\
\hline A32 & 203230.3 & 403433.0 & 7.038 & 0.554 & 0.312 & \\
\hline A33 & 203234.9 & 405239.0 & 8.598 & 0.565 & 0.305 & \\
\hline A34 & 203136.9 & 42121.0 & 6.653 & 0.378 & 0.305 & \\
\hline A35 & 203055.5 & 405454.0 & 8.471 & 0.506 & 0.305 & \\
\hline A36 & 203458.7 & 413617.0 & 6.36 & 0.544 & 0.302 & \# 357 [7] \\
\hline A37 & 20364.5 & 405613.0 & 7.675 & 0.614 & 0.292 & \\
\hline A38 & 203234.8 & 405617.0 & 8.564 & 0.553 & 0.292 & KMJ 1315 [6] \\
\hline A39 & 203227.3 & 405518.0 & 7.857 & 0.492 & 0.285 & KMJ 1834 [6] \\
\hline $\mathrm{A} 40$ & 203513.6 & 405525.0 & 8.391 & 0.601 & 0.284 & \\
\hline $\mathrm{A} 41$ & 20318.3 & 42242.0 & 7.029 & 0.517 & 0.269 & \\
\hline A 42 & 202957. & 41953.0 & 8.444 & 0.439 & 0.266 & \\
\hline A 43 & 203238.5 & 412513.0 & 7.699 & 0.306 & 0.222 & \\
\hline $\mathrm{A} 44$ & 203146 . & 404324.0 & 8.262 & 0.46 & 0.211 & \\
\hline A 45 & 202946.6 & 4158.0 & 8.455 & 0.425 & 0.192 & \\
\hline A46 & 20310.1 & 404949.0 & 7.838 & 0.39 & 0.178 & \\
\hline
\end{tabular}

Notes: [1] Griffith et al. (1991); [2] Vaiana et al. (1981); [3] Massey \& Thompson (1991); [4] Parthasarathy \& Jain (1995); [5] McDowell (1994); [6] Kobulnicky et al. (1994); [7] Reddish et al. (1966).

relationship to the far-infrared and the radiocontinuum sources deserve further study. The spectral type B2 assigned by Miralles et al. is derived under the assumption that all the ionizing photons of the stars are used in ionizing the nebula neglecting the presence of dust, and is thus a lower limit.

\subsection{Stars with $\mathrm{CO}$ in emission}

While none of the Br $\gamma$-emitting stars in our sample displays detectable $\mathrm{CO}$ overtone emission that is sometimes observed in $\mathrm{B}[\mathrm{e}], \mathrm{Be}$, and $\mathrm{LBV}$ stars, three objects in our sample do show $\mathrm{CO}$ in emission, as shown in Fig. 9 
Table 2. Observed stars with $\operatorname{Br} \gamma$ in emission.

\begin{tabular}{rcccccc}
\hline \hline Number & $\alpha(2000)$ & $\delta(2000)$ & $K$ & $(J-H)$ & $(H-K)$ & remarks \\
\hline B1 & 20367.5 & 41409.3 & 8.893 & 4.229 & 3.1 & possibly associated to IRAS 20343+4129 [8] \\
B2 & 203413.3 & 41814.0 & 8.534 & 2.977 & 2.455 & \\
B3 & 203430.9 & 413039.1 & 7.288 & 3.439 & 2.413 & possibly associated to IRAS 20327+4120 \\
B4 & 203447. & 405448.6 & 8.887 & 2.264 & 1.653 & \\
B5 & 20342.9 & 411040.9 & 9.982 & 2.036 & 1.432 & \\
B6 & 203211.3 & 404034.0 & 8.586 & 1.701 & 1.427 & possibly associated to IRAS 20303+4030 \\
B7 & 203413.4 & 41157.8 & 9.893 & 1.96 & 1.397 & \\
B8 & 203325.6 & 41831.5 & 9.667 & 1.579 & 1.307 & \\
B9 & 203516.3 & 411236.2 & 9.177 & 1.145 & 0.736 & \\
B10 & 203327.1 & 413558.5 & 8.177 & 0.777 & 0.67 & \# 285 [2] \\
B11 & 203318.5 & 411535.2 & 7.952 & 0.862 & 0.623 & V2188 Cyg [9]; [3] \\
B12 & 203230.9 & 41100.6 & 8.692 & 0.852 & 0.598 & \\
B13 & 203334.3 & 411811.4 & 7.826 & 0.723 & 0.585 & \# 708; B1.5V Herbig Ae/Be [12] \\
B14 & 20345.6 & 405219.6 & 8.91 & 0.852 & 0.532 & \\
B15 & 203318.7 & 405937.0 & 8.127 & 0.657 & 0.457 & \\
B16 & 203443.5 & 41294.0 & 7.718 & 0.516 & 0.409 & \# 360, B1.5III [2] \\
B17 & 203027.3 & 411325.0 & 6.443 & 0.804 & 0.406 & \\
B18 & 203457.8 & 414354.0 & 7.42 & 0.672 & 0.365 & \\
B19 & 203310.7 & 41158.0 & 5.556 & 0.611 & 0.332 & VI Cyg 9: O5Iab:e... [11] \\
B20 & 20348.5 & 413659.0 & 5.993 & 0.443 & 0.236 & VI Cyg 11: O6e [11] \\
\hline
\end{tabular}

Notes: same as in Table 1, plus: [8] Miralles et al. (1994); [9] Pigulski \& Kolaczkowski (1998); [10] Thé et al. (1994); [11] Herrero et al. (1999).

Table 3. Equivalent widths of emission lines (in $\AA$ ).

\begin{tabular}{|c|c|c|c|c|c|c|c|c|c|c|c|}
\hline Number & $\begin{array}{c}\mathrm{Br} \gamma \\
2.166\end{array}$ & $\begin{array}{l}\operatorname{Br} 16 \\
1.555\end{array}$ & $\begin{array}{l}\operatorname{Br} 15 \\
1.569\end{array}$ & $\begin{array}{l}\text { Br14 } \\
1.588\end{array}$ & $\begin{array}{l}\text { Br13 } \\
1.611\end{array}$ & $\begin{array}{c}\text { Br12 } \\
1.641\end{array}$ & $\begin{array}{c}\text { Br11 } \\
1.681\end{array}$ & $\begin{array}{l}\text { Br10 } \\
1.736\end{array}$ & $\begin{array}{c}\text { HeI } \\
1.700\end{array}$ & $\begin{array}{c}\mathrm{HeI} \\
2.058\end{array}$ & 2.225 \\
\hline B1 & 3.3 & & & & & & & & & & 7.3 \\
\hline B2 & 6.3 & & & & & & & & & & \\
\hline B3 & 17.2 & & & & & 6.8 & 13.4 & 16.6 & & & \\
\hline B4 & 10.6 & & & & & & & & & & \\
\hline B5 & 8.9 & & & & & & & & & & \\
\hline B6 & 10.3 & & & & & & & 9.9 & & & \\
\hline B7 & 12.2 & & & & & & & & & & \\
\hline B8 & 10.9 & & & & & & & & & & \\
\hline B9 & 27.0 & 2.7 & 4.8 & 12.6 & 15.3 & 16.6 & 16.3 & 19.4 & & & \\
\hline B10 & 29.6 & 5.7 & 8.7 & 14.0 & 17.3 & 17.1 & 19.2 & 21.3 & & 8.4 & \\
\hline B11 & 22.2 & 3.8 & 6.7 & 11.7 & 9.1 & 14.9 & 15.9 & 14.8 & & & \\
\hline $\mathrm{B} 12$ & 22.0 & & & 6.5 & 7.5 & 9.8 & 10.0 & 9.4 & & 3.1 & \\
\hline B13 & 13.5 & 1.6 & 3.8 & 7.1 & 8.9 & 10.1 & 12.4 & 12.4 & & 4.2 & \\
\hline B14 & 23.2 & & & & & & & 10.6 & & 18.8 & \\
\hline B15 & 9.9 & & & 4.7 & 7.6 & 6.3 & 12.6 & 16.1 & & 4.4 & \\
\hline B16 & 23.7 & & & 11.3 & 13.2 & 13.7 & 15.3 & 15.1 & 4.4 & & \\
\hline B17 & 13.4 & & & & & & & 6.9 & 7.0 & & \\
\hline B18 & 17.1 & & & & & & & & & & \\
\hline B19 & 6.2 & & & & & & & & & & \\
\hline B20 & 8.2 & & & & & & & & & & \\
\hline
\end{tabular}

and listed in Table 4. These objects are reddened by widely different amounts and only one of them, C3 in Table 3, is associated with an object identified in the visible, Cyg OB2 \#1071.
The spectrum of $\mathrm{C} 1$ displays strong emission at $2.12 \mu \mathrm{m}$, besides the $\mathrm{CO}$ emission. Intense emission near that wavelength is often seen in the spectra of evolved massive stars due to HeI. However, due to the absence 


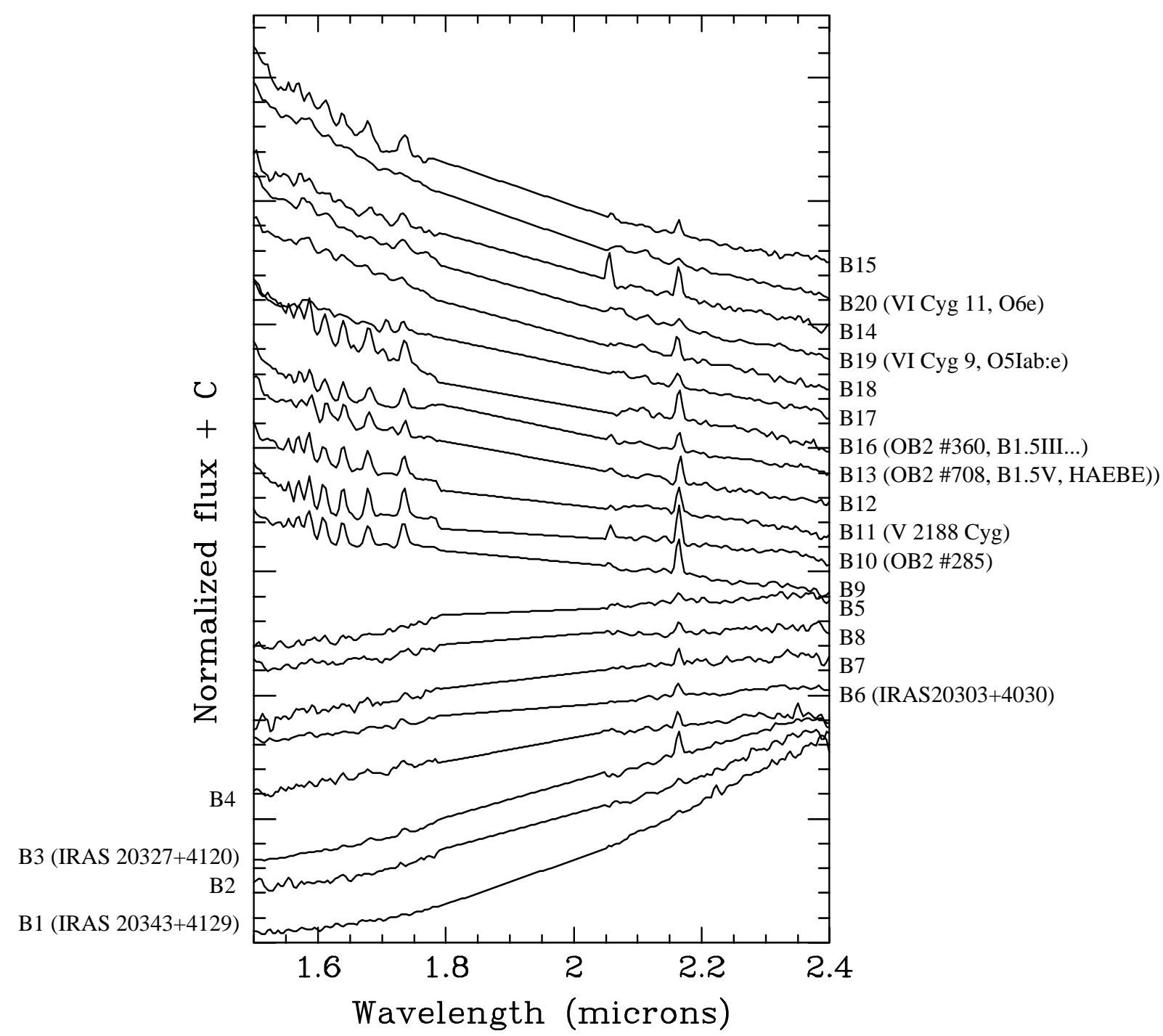

Fig. 7. Stars with $\operatorname{Br} \gamma$ seen in emission in our spectra, arranged by order of increasing $(H-K)$ according to 2 MASS data. This plot includes both objects previously observed by other authors as well as new ones. The spectra are normalized to the flux in the $2.1-2.2 \mu \mathrm{m}$ interval.

Table 4. Observed stars with CO in emission.

\begin{tabular}{rcccccl}
\hline Number & $\alpha(2000)$ & $\delta(2000)$ & $K$ & $(J-H)$ & $(H-K)$ & remarks \\
\hline C1 & 203443.3 & 405313.7 & 9.342 & 2.954 & 2.146 & probably associated to IRAS 20328+4042 \\
& & & & & and radio continuum source [12] \\
C2 & 203231.2 & 411444.1 & 9.814 & 1.409 & 1.186 & \\
C3 & 203410.4 & 41248.0 & 9.417 & 0.897 & 0.862 & {$[3]$} \\
\hline
\end{tabular}

Notes: same as in Table 1, plus: [12] Wendker et al. 1991, A\&A, 241, 551.

of other helium lines and of $\operatorname{Br} \gamma$, together with the simultaneous existence of strong CO emission, we are inclined to attribute the emission at that wavelength in $\mathrm{C} 1$ to $\mathrm{H}_{2}$ instead, with both $\mathrm{H}_{2}$ and $\mathrm{CO}$ indicating the presence of a massive circumstellar envelope or disk. The presence of important amounts of molecular gas and dust around this object appears to be confirmed by the likely association of C1 with IRAS $20328+4042$, although the offset between the coordinates of both sources, $29^{\prime \prime}$, is only marginally within the IRAS error ellipse. This offset may be explained by the existence of small extended emission around $\mathrm{C} 1$, clearly visible in the $K$-band image of the field presented in Fig. 10. The arc-shaped nebulosity around $\mathrm{C} 1$ is suggestive of dynamical interaction with 


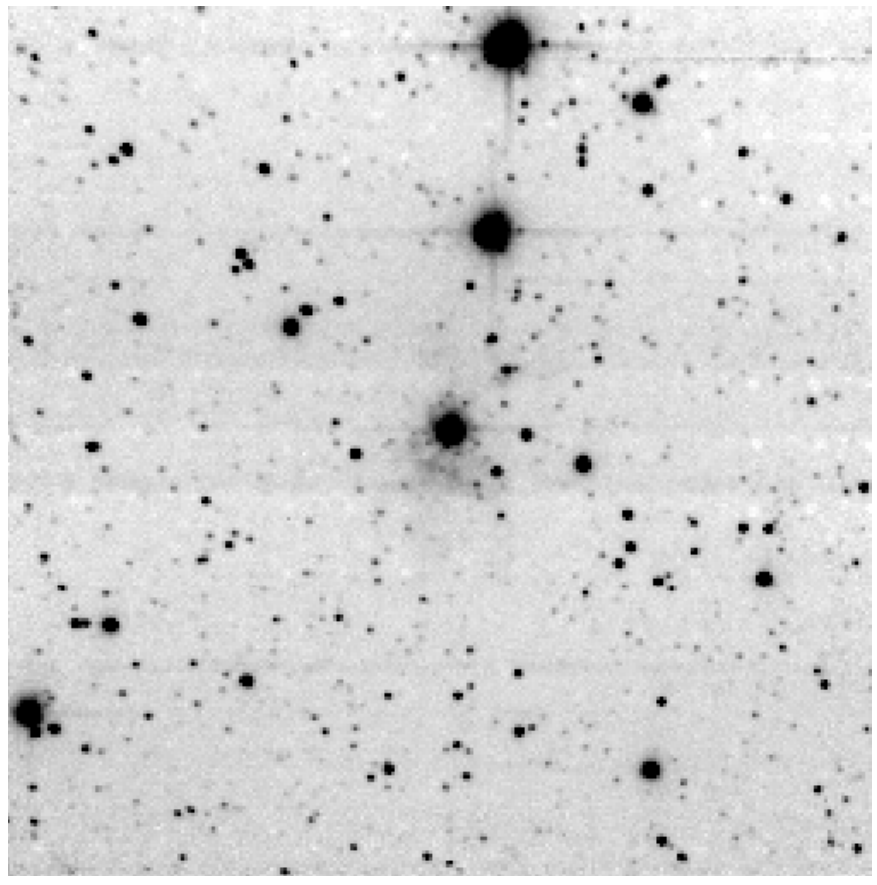

Fig. 8. $K$-band image of the nebula associated to the emissionline star B3 (at the center). The field of view is $5^{\prime} \times 5^{\prime}$, with North at the top and East to the left.

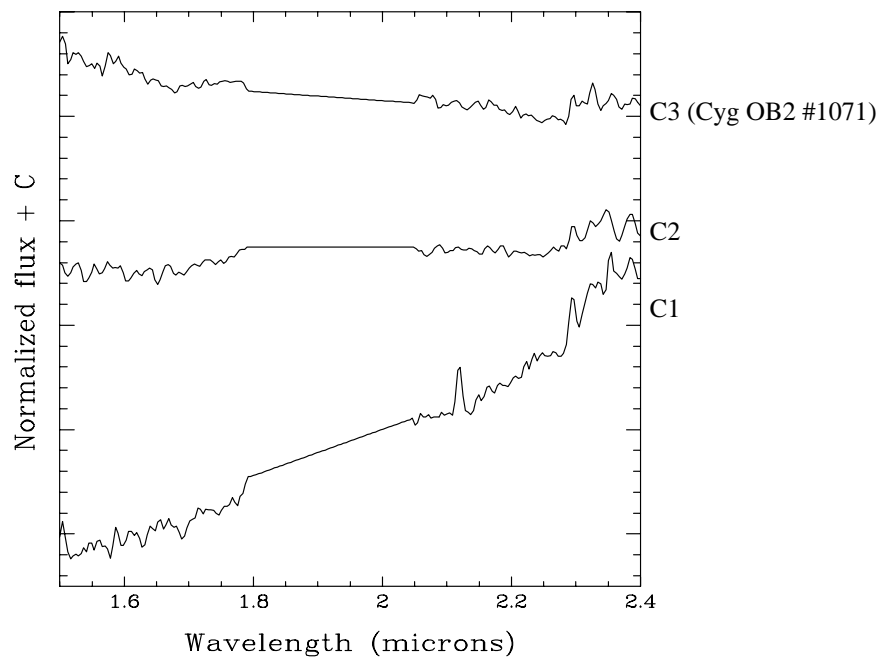

Fig. 9. Stars with CO seen in emission in our spectra, arranged by order of increasing $(H-K)$ according to 2 MASS data. The spectra are normalized to the flux in the $2.1-2.2 \mu \mathrm{m}$ interval.

the surrounding medium. Faint resolved emission in the $21 \mathrm{~cm}$ continuum is also observed at the position of $\mathrm{C} 1$ (Wendker et al. 1991).

The other two objects in our survey showing CO overtone emission do not display any other emission features in our spectra nor are associated with either radio continuum or mid-infrared sources. Their relatively blue spectral energy distributions suggest that they are emerged objects whose CO emission may be due to a moderately massive circumstellar disk.

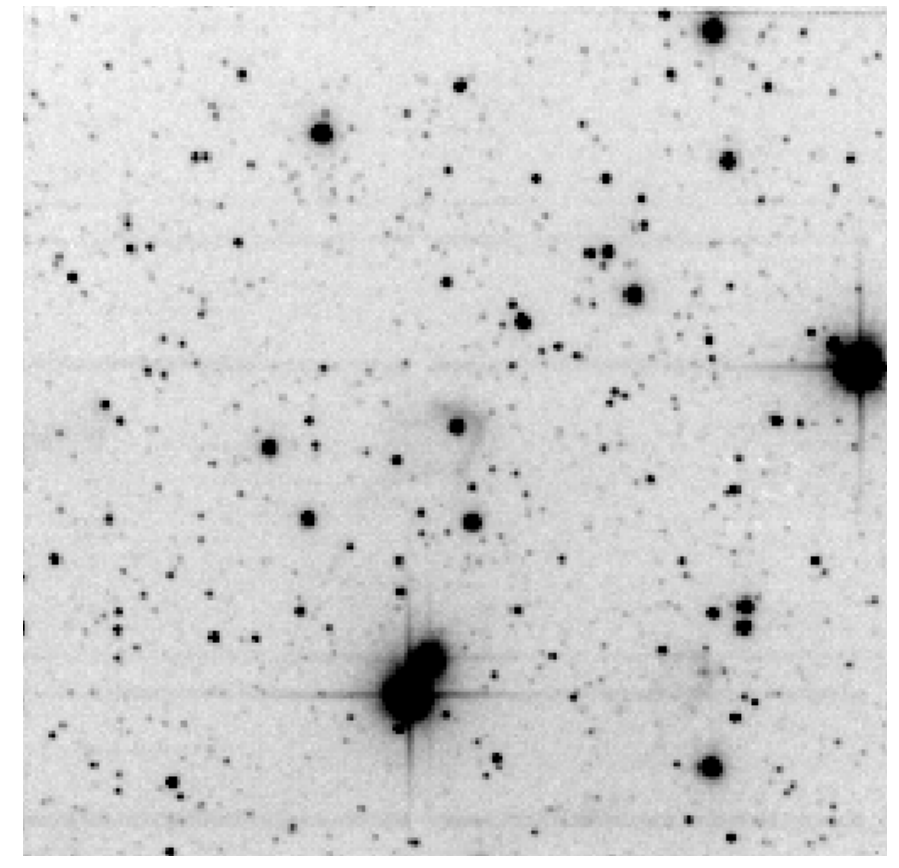

Fig. 10. The field around star C1, which is the object at the center of the image with an arc-shaped nebula towards the West (center). North is at the top. The field of view shown measures $5^{\prime} \times 5^{\prime}$.

\subsection{Possible red supergiants}

The considerable number of evolved massive stars that we find in our sample suggests that red supergiants may also be present in Cygnus OB2. Our selection criteria described in Sect. 3 is not designed to detect these stars, but rather tends to actually exclude them, as red supergiants should lie along the upper reddening band in the color-color diagram. However, the fact that for $(H-K)>0.5$ we included many stars already in that band as demonstrated by the large number of stars with cool near-infrared spectra that we encounter, has prompted us to have a detailed look at those searching for possible supergiants belonging to the association.

The $K$-band spectra of late-type supergiants are characterized by strong $\mathrm{CO}$ absorption bands whose depth is matched only by late-type Miras (Lançon \& Wood 2000). However, unlike the latter, late-M supergiants do not display the prominent water vapour bands that form in the extended atmospheres of Miras. We have thus used the approach proposed by Comerón et al. (in preparation) to select late-M supergiants based on the strengths of these molecular features, based on the following spectrophotometric bands:

$-f_{\mathrm{CO}}^{L}: 2.29 \mu \mathrm{m} \rightarrow 2.40 \mu \mathrm{m}$ (flux in the region of the CO bands).

- $f_{\mathrm{CO}}^{C}: 2.17 \mu \mathrm{m} \rightarrow 2.28 \mu \mathrm{m}$ (flux in the $K$-band continuum, medium passband).

$-f_{H}^{L}: 1.73 \mu \mathrm{m} \rightarrow 1.78 \mu \mathrm{m}$ (flux in the blue wing of the water vapour band). 
$-f_{H}^{C}: 1.65 \mu \mathrm{m} \rightarrow 1.70 \mu \mathrm{m}$ (flux in the $H$-band continuum).

$-f_{K}^{L}: 2.05 \mu \mathrm{m} \rightarrow 2.10 \mu \mathrm{m}$ (flux in the red wing of the water vapour band).

- $f_{K}^{C}: 2.23 \mu \mathrm{m} \rightarrow 2.28 \mu \mathrm{m}$ (flux in the $K$-band continuum, narrow passband).

The extinction at $K$ is then estimated as

$$
A_{K}=1.78\left(0.848-2.5 \log \frac{f_{H}^{C}}{f_{K}^{C}}\right)
$$

where use is made of the Rieke \& Lebofsky (1985) extinction law and an intrinsic color $(H-K)_{0}=0.30$ is assumed for M supergiants (Tokunaga 2000). The following indices measuring the strength of the $\mathrm{CO}$ and $\mathrm{H}_{2} \mathrm{O}$ features are then approximately reddening-independent:

$$
\begin{aligned}
& {[\mathrm{CO}]_{\text {spec }}=-2.5 \log \frac{f_{\mathrm{CO}}^{L}}{f_{\mathrm{CO}}^{C}}+0.085 A_{K}} \\
& {\left[\mathrm{H}_{2} \mathrm{O}\right]_{\text {spec }}=-2.5 \log \frac{f_{H}^{L}}{f_{H}^{C}}-2.081 \log \frac{f_{K}^{L}}{f_{K}^{C}} .}
\end{aligned}
$$

Late-M supergiants are expected to occupy the region of the $[\mathrm{CO}]_{\text {spec }},\left[\mathrm{H}_{2} \mathrm{O}\right]_{\text {spec }}$ diagram characterized by the highest values of $[\mathrm{CO}]_{\text {spec }}$ together with very low values of $\left[\mathrm{H}_{2} \mathrm{O}\right]_{\text {spec }}$. To assess whether or not the 14 possible red supergiants that we find in this way do belong to Cygnus OB2, we have estimated their absolute magnitudes assuming that they are at the distance of the association and using the estimated extinction as given by Eq. (2). The values that we find in this way range between $M_{K}=-3.6$ and $M_{K}=-6.8$, thus being in all cases several magnitudes fainter than typical late-type supergiants (e.g. Elias et al. 1985) and leading to the conclusion that they are most likely background stars. The relatively large number of such objects found in the direction of Cygnus OB2 is not surprising in view of the depth of our sample for such infrared-bright objects: for an absolute magnitude $M_{K}=-10$, and even assuming $A_{V}=30$, our magnitude limit reaches up to $25 \mathrm{kpc}$ from the Sun, thus probing essentially the entire line of sight up to the edge of the disk of the Galaxy in that direction, and in particular a large volume across the Perseus arm.

Let us finally stress the fact already mentioned at the beginning that our failure to identify any red supergiants in Cygnus OB2 does not imply that such stars are absent from the association, since our color-based selection criterion is biased against such objects.

\subsection{Color-color and color-magnitude diagrams of candidate members of Cygnus OB2}

Figures 11 and 12 show the positions occupied in colorcolor and color-magnitude diagrams of all spectroscopically observed members of the association. In Fig. 11, nearly all the early-type stars with $H-K<0.5$ are well aligned in a band parallel to the reddening vector but offset by $\Delta(J-H) \simeq 0.1$ with respect to it. This may be

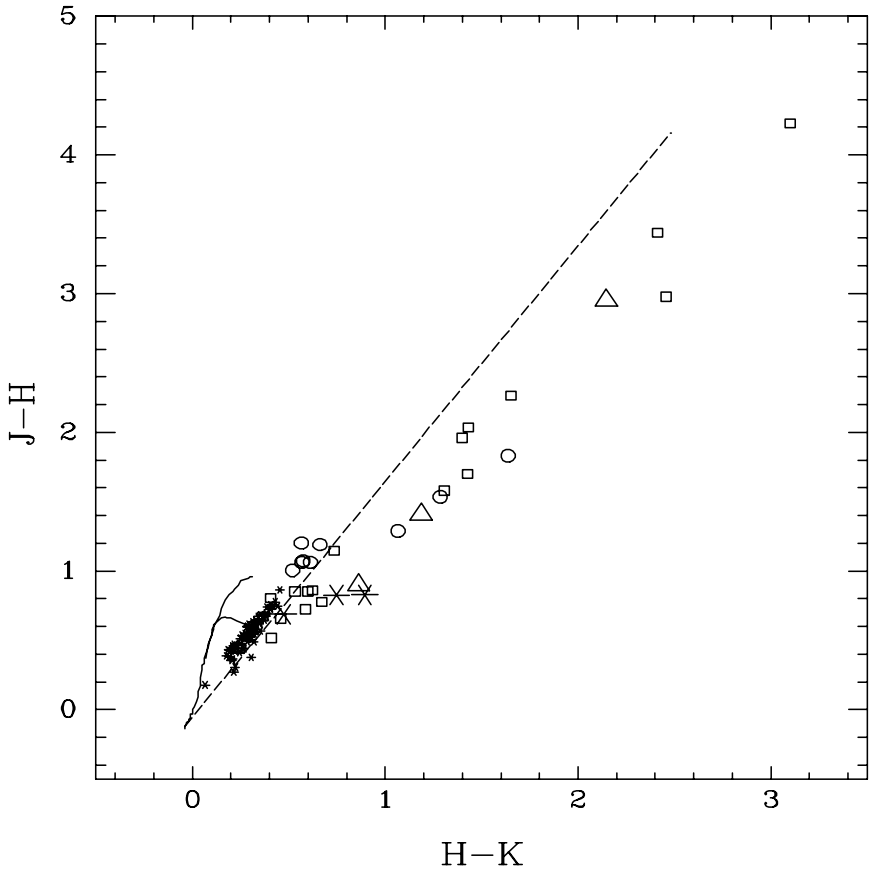

Fig. 11. Color-color diagram of all the objects observed. Small asterisks, blue early-type stars $(H-K<0.5)$; circles, reddened early-type stars $(H-K>0.5)$; squares, stars with $\mathrm{Br} \gamma$ in emission; triangles, stars with CO in emission; large asterisks, WR stars. The dashed line is the locus occupied by reddened B0 stars with intrinsic colors $J-H=-0.12, H-K=-0.04$, corresponding to a B0 star according to Tokunaga (2000); the end of the line corresponds to an extinction of $A_{V}=40$.

indicative of a small incorrectness in the adopted intrinsic color calibration, as many of these stars are known to have earlier spectral types and hence colors bluer than those of a B0 star, where we have placed the origin of the reddening vector. Most of the newly found early-type stars with featureless spectra but redder colors are found in the extension of the same line.

The vast majority of the emission-line stars, as well as the stars with $\mathrm{CO}$ emission, the three WR stars, and three of the stars classified as having non-emission, earlytype spectra, are found to the right of the reddening vector, indicating the existence of significant amounts of dust emission in their surroundings. The range of extinctions is difficult to assess precisely, as this would need the disentangling of the contribution of the circumstellar emission and the foreground extinction to the position in the diagram. However, the fact that the reddest objects tend to display spectroscopic signatures of either strong winds or excitation of molecular gas suggests that the origin of the red colors is largely contributed by extinction produced by dust around the star, either in a stellar wind-blown nebula or in the material left over from the formation of the star.

Figure 12 shows that the three reddest emission-line stars that we observed have $K$ magnitudes placing them among the brightest members of Cygnus OB2 if they were dereddened along the extinction vector. This is actually an upper limit to their intrinsic brightness, because as we 


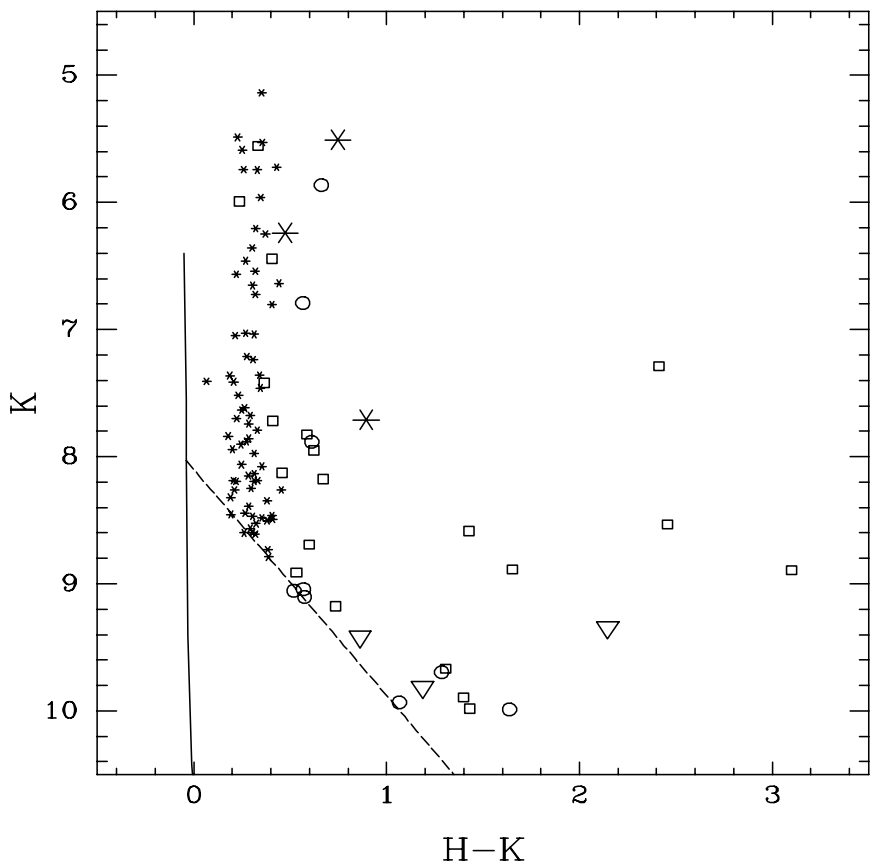

Fig. 12. Color-magnitude diagram of all the objects observed. Symbols as in Fig. 11. The dashed line is the locus occupied by reddened B0 stars at the adopted distance of Cygnus OB2, like in Fig. 1.

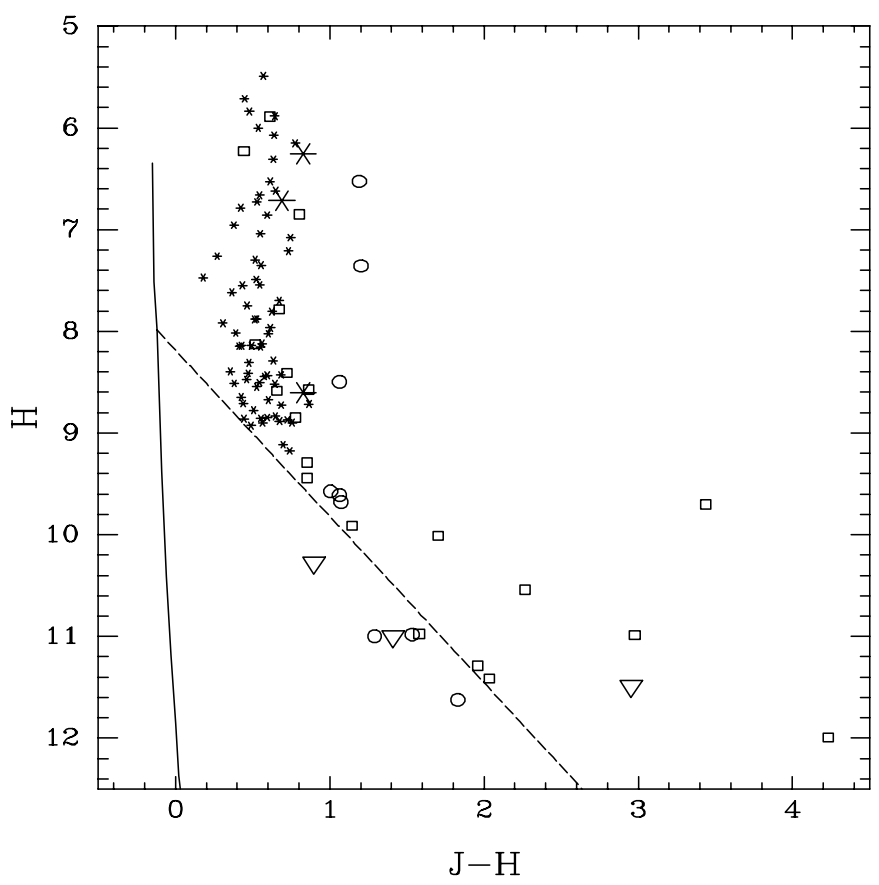

Fig. 13. Color-magnitude diagram of all the objects observed, now considering the $J$ and $H$ magnitudes to reduce the influence of circumstellar emission. Symbols as in Fig. 11. The dashed line is the locus occupied by reddened B0 stars at the adopted distance of Cygnus OB2, like in Fig. 1.

have argued considerable contribution to the flux by circumstellar material may be expected. However, Fig. 13 shows that this is still true when the $H,(J-H)$ diagram, which should be less affected by circumstellar emission,

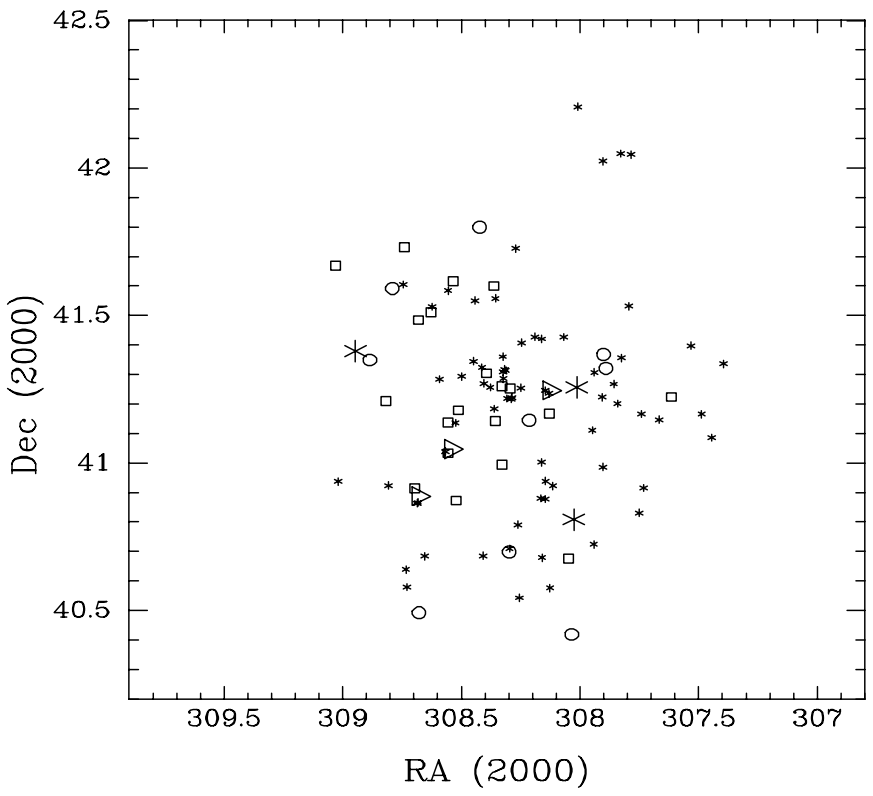

Fig. 14. Positions of all the early-type and related stars observed in the present study. Symbols as in Fig. 11.

is considered. Sources B1, B2, and B3 thus appear as especially interesting objects for further studies of the most massive and luminous objects in Cygnus OB2, particularly in view to clarify their relationship with their associated IRAS sources in the cases of B1 and B3.

\subsection{Spatial distribution}

The nearly extinction-free view of the Cygnus OB2 region provided by our infrared-selected sample yields a much more unbiased picture of the extent of the association than available from a census of members identified in the visible. As pointed out by Knödlseder (2000), the true shape of Cygnus OB2 emerging from infrared star counts seems to be much rounder than that derived from visible surveys, the latter being dominated by an irregular distribution of the extinction in front of the association.

The positions of all the known and new candidate members of Cygnus OB2 that we observed are plotted in Fig. 14. Since our sample is restricted to candidate O-type stars and thus has a much brighter limit than the sample considered by Knödlseder, our distribution of points in Fig. 14 is far more sparse than that leading to the map shown in Fig. 5 of Knödlseder. Our restriction to a radius of one degree from the center of the association also may leave outside some of the outermost regions of Cygnus OB2. However, our results already seem to confirm the deviation from circular symmetry found by Knödlseder. We note in particular the scarcity of members towards the East and the North of the center of the association, while the larger extension towards the Southwestern quadrant, most probably reaching beyond the boundaries of the one-degree radius selected in our study, is obvious.

A comparison among the spatial distributions of the different types of massive stars considered here shows some 
possible intriguing differences. The concentration of bluer early-type stars $(H-K<0.5)$ towards the Southwestern quadrant is more pronounced, as may be expected as a consequence of the somewhat higher extinction towards the East and Northeast. However, no obvious asymmetry is seen in the distribution of emission-line stars, which are slightly more represented in the Northeastern quadrant than in the Southwestern one. We should refrain from making a definitive statement on the reality of this difference, as the low number of emission-line stars makes the statistical significance of this difference rather limited. It may be tempting to speculate that the scarcity of emission-line (e.g. evolved) massive stars with respect to normal $\mathrm{O}$ and early B stars in the Southwestern quadrant of the association may reflect a younger average age of that region, perhaps as consequence of the progression of star formation in that direction. However, the small number of stars on which this conjecture is based makes it only tentative, and we mention it here just as a possible direction for future observations.

\subsection{The O-star contents of Cygnus OB2}

The rich contents of Cygnus OB2 in O-type stars, or in stars having evolved from a O-type progenitor, has been known since the earliest studies on this association, and has been quantified more recently by several authors (Massey \& Thompson 1991; Massey et al. 1995; Parthasarathy \& Jain 1995; Knödlseder 2000) who have investigated the upper end of its mass function on the basis of members identified either in the visible or the infrared. An important driver of recent investigations on Cygnus OB2 has been the recognition of its importance in linking the emission of ${ }^{26} \mathrm{Al}$ observed in the Cygnus region to nucleosynthesis processes in the interior of the massive stars of the association (del Río et al. 1996; Cerviño et al. 2000; Knödlseder 1999), within the broader goal of understanding the contribution of $\mathrm{OB}$ associations to the chemical enrichment processes in galactic disks. With a different scope, Comerón et al. (1998) have considered the energetic activity of the most massive stars in Cygnus OB2 as a possible trigger of star formation in its neighbourhood, reflected nowadays in the peculiar motions of stars in other associations surrounding it.

It is thus interesting to estimate the O-star contents of Cygnus OB2 in the light of our results, and to compare it to the most recent estimate of Knödlseder (2000), as both should be nearly free from extinction biases. Let us start by considering the 77 early-type stars that we have observed: of these, 31 have been spectroscopically classified in the visible and 24 have been found to be O-type, as described in Sect. 4.1, the other 7 belonging to B type. Assuming that the same ratio holds for the entire set of 77 stars, we thus estimate 60 O-type stars among those.

As described in Sect. 4.2, the stars with $\operatorname{Br} \gamma$ in emission are expected to represent different stages of the evoution of the most massive stars. Assuming that all these stars started their lives as O stars, this adds 20 more stars. This may not be the case for all these objects however, especially for the stars closer to the B0 limit in Fig. 12, given the infrared excess commonly displayed by these objects: Fig. 13 shows that one of these stars is slightly fainter than the B0 limit in the $H,(J-H)$ diagram, and five more are very close to it, so the actual number of evolved massive stars with O-type progenitors may be somewhere between 14 and 20. Other stars that obviously have evolved from an O-type phase are the three WR stars, thus raising the census to $76-83$ objects. We note that the spectral characteristics of these 23 emissionline stars, of which 16 have been identified as such for the first time in the present work, indicate strong mass loss rates, thus being important for the present-day mechanical energy input in the association.

We finally consider the three stars with CO emission, in which circumstellar material is also expected to contribute to the luminosity. Two of them are close to the B0 boundary, and fall well below it in the $H,(J-H)$ diagram of Fig. 13; their brightness at $K$ is thus likely to be largely due to circumstellar emission, and we consider it unlikely that they are related to O-type stars. While the circumstellar contribution to the flux at $K$ is also important for the third one, $\mathrm{C} 1$, its high luminosity also in $H$ leads us to include it in our census of O-type stars.

We thus estimate that we have identified between 77 and 84 O-type stars or closely related objects in the association. This may not be a complete census however, mainly due to two reasons. First, as Fig. 5 suggests, our observations do not completely sample the band of lightly reddened $(H-K<0.5)$ early-type stars; there are 23 objects along this band for which the available time did not allow us to obtain spectra. Fortunately, spectral classifications are found in the literature for 14 of them, mostly in Massey \& Thompson (1991) but also from Herrero et al. (1999) and Walborn \& Howarth (2000, for the remarkable O3Iab star VI Cyg 7). Of these, 10 are classified as O-type stars, one is a B1Ib star, and three are classified as F- and G-type and thus likely foreground. Assuming that similar ratios apply for the other 9 stars without spectral classification, this makes us estimate that there are about 16 stars in thus group. A second source of incompleteness may be the extension of the association beyond our onedegree-radius limit, especially in the Southeastern direction. The stellar density contours presented by Knödlseder (2000) do not show any significant enhancement in those regions. However, we should keep in mind that even a low density halo might contain a non-negligible fraction of the members of Cygnus OB2, given the quadratic growth of projected area with radius.

Our overall estimate on the total number of O-type or related stars in Cygnus OB2 is thus 90-100, slightly below Knödlseder's estimate but well compatible within uncertainties. Of these, approximately $25 \%$ display emission lines in our spectra (including possible $\mathrm{Br} \gamma$-emitting stars among the non-observed part of the bluer sample). The confirmation of Knödlseder's estimate at the highest 
masses support his conclusions on the total stellar contents and reaffirms Cygnus OB2 as one of the most massive stellar associations in our Galaxy, perhaps comparable to a young globular cluster as claimed by that author.

\section{Summary and conclusions}

In this paper we have discussed the results of a spectroscopic survey in the $H$ and $K$ bands of stars in the direction of Cygnus OB2, within a radius of one degree from its nucleus. The sample was selected on the basis of their $J H K$ photometry as published in the Second Incremental Release of the 2MASS Point Source Catalog. Our selection is based on the fact that the color-color diagram of Cygnus OB2 shows two clearly distinct sequences, both parallel to the reddening vector: the lower one (i.e., bluer $J-H$ at a given $H-K$ ) corresponds to early-type, moderately reddened members of the association, while the upper and most populated one corresponds to background, reddened giants. Some objects appear on or near the lower reddening sequence and large values of $J-H$ and $H-K$ and may be obscured or embedded members of Cygnus OB2.

We obtained spectra for stars brighter than $K=10$ near the lower sequence and with a position in the $K$, $(H-K)$ diagram consistent with them being O-type stars. The low resolution and moderate signal-to-noise ratio of our spectra does not allow an accurate spectral classification, but suffices to distinguish between the featureless spectra of early-type stars and those of cool stars, displaying a different continuum shape and prominent molecular absorption bands due to $\mathrm{CO}$ and $\mathrm{H}_{2} \mathrm{O}$. Intermediate-type stars should be too faint at the distance of Cygnus OB2 to be included in our target list.

We have identified 77 stars that we tentatively classify as early-type based on the shape of the continuum and the lack of visible features in our spectra. Only 31 have been previously classified based on visible spectra, 24 of them being O-type and 7 early B-type. Assuming that the same O- to B- star ratio holds for all the 77 stars, we thus estimate that there are approximately $60 \mathrm{O}$ stars in this sample.

We also identify 20 stars with $\operatorname{Br} \gamma$ emission in their $K$-band spectra, and in many cases other lines (mainly of $\mathrm{H}$ and $\mathrm{He}$ ) in emission as well. These features are typical of the spectra of evolved massive stars undergoing intense mass loss in stellar winds. Of these, 16 are identified as emission-line stars for the first time in this study. Most of these stars are highly reddened, with 8 of them having $H-K>1$ and reaching up to 3.1. Most of these stars, and all those having $H-K>1$, appear to the right of the reddening vector, indicating the existence of circumstellar emission at long wavelengths. Based on the $J H K$ colors and fluxes, three of these stars, unidentified so far, seem to be among the most luminous members of Cygnus OB2. Three additional bright red stars showing $\mathrm{CO}$ overtone and infrared excess emission (one also showing $\mathrm{H}_{2} \mathrm{~S}(1)$ emission at $2.12 \mu \mathrm{m}$ ) are also identified.
Finally, we consider the possible existence of red supergiant members of Cygnus OB2 in our sample. Although we do find several stars with infrared spectra suggestive of them being supergiants, they are all too faint to be members of Cygnus OB2. This does not rule out the possibility that Cygnus OB2 does contain late-type supergiants, as our target selection is biased against these stars.

The location of the Cygnus OB2 members identified in this study, both the new ones and the previously known ones, confirm the shape of Cygnus OB2 as being roughly circular with an extension towards the Southwest, as determined by Knödlseder (2000) using star counts. We point out a possible difference in the distribution of normal early-type stars and that of emission-line stars, the latter having a more regular distribution around the core of the association, although the statistical significance of this result is limited by the small number of objects on which it is based.

Taking into account our results, those of other studies that have provided spectral classifications in the visible, and approximate corrections for incompleteness, we estimate that Cygnus OB2 contains between 90 and 100 O-type stars or stars having evolved from a O-type progenitor, in very good agreement with recent estimates by Knödlseder (2000). Of these, approximately $25 \%$ have emission lines in their infrared spectra.

Since its discovery over 40 years ago Cygnus OB2 has been recognized as an outstanding association, with new studies only confirming or enhancing its richness. While photometric and spectroscopic surveys have been hampered by the extinction hiding from view a significant part of the association, recent efforts based on the 2MASS nearinfrared survey are providing an accurate estimate of the massive star contents of Cygnus OB2. The good agreement found between the independent approaches followed by Knödlseder (2000) and the present work indicates that the census of massive stars in Cygnus OB2 is nearing completion now, at least as far as the region within one degree from the center of the association is concerned. Such a complete census of by far the richest OB association in the solar neighbourhood, and probably one of the richest in our Galaxy, presents us with a unique opportunity to study in close-up detail its entire high-mass end and the wide variety of phenomena associated to the activity of very massive stars. High resolution infrared spectroscopy at high signal-to-noise ratio of all the objects listed in this study, as well as visible spectroscopy of the new, less reddened members, should provide a most valuable observational resource for understanding the formation, evolution, and impact on their environments of large OB associations in galactic disks, as well as the complex spectral variety resulting from the late evolutionary stages of the most massive stars.

Acknowledgements. We warmly thank the organizers of the first NEON Summer School, especially Dr. Michel Dennefeld, for their highly successful effort in making it a very profitful scientific and learning experience. The rest of participants and 
faculty of the School are also thanked for having made possible the unique human atmosphere during the two weeks of the School. The NEON Summer Schools are funded by the European Union. We also acknowledge the generous allocation of time at the Calar Alto $1.23 \mathrm{~m}$ telescope in the summer of 2001, which allowed us to follow up on this project and obtain the considerable number of spectra discussed in this paper. Constructive comments by an anonymous referee are thanked as well. Finally, it is a great pleasure to thank all the staff of the Calar Alto Observatory, and especially Mr. Santos Pedraz, for their support in our observing runs. This research has made use of the SIMBAD database, operated at CDS, Strasbourg, France, as well as of data products from the Two Micron All Sky Survey, which is a joint project of the University of Massachusetts and the Infrared Processing and Analysis Center/California Institute of Technology, funded by the National Aeronautics and Space Administration and the National Science Foundation.

\section{References}

Cerviño, M., Knödlseder, J., Schaerer, D., von Ballmoos, P., \& Meynet, G. 2000, A\&A, 363, 970

Comerón, F., Torra, J., \& Gómez, A. E. 1998, A\&A, 330, 975

Comerón, F., \& Torra, J. 2001, A\&A, 375, 539

Dobashi, K., Bernard, J.-P., \& Fukui, Y. 1996, ApJ, 466, 282

Drilling, J. S., \& Landolt, A. U. 2000, in Allen's Astrophysical Quantities, ed. A. N. Cox (AIP Press)

Elias, J. H., Frogel, J. A., \& Humphreys, R. M. 1985, ApJS, 57,91

Glass, I. S., Whitelock, P. A., Catchpole, R. M., \& Feast, M. W. 1995, MNRAS, 273, 383

Griffith, M., Langston, G., Heflin, M., Conner, S., \& Burke, B. 1991, ApJS, 75, 801

Hanson, M. M., Conti, P. S., \& Rieke, M. J. 1996, ApJS, 107, 281

Herrero, A., Corral, L. J., Villamariz, M. R., \& Martín, E. L. 1999, A\&A, 348, 542

Johnson, H. L., \& Borgman, J. 1963, Bull. Astron. Inst. Netherlands, 17, 115

Johnson, H. L., \& Morgan, W. W. 1954, ApJ, 119, 344
Knödlseder, J. 1999, ApJ, 510, 915

Knödlseder, J. 2000, A\&A, 360, 539

Kobulnicky, H. A., Molnar, L. A., \& Jones, T. J. 1994, AJ, 107, 1433

Lançon, A., \& Wood, P. R. 2000, A\&AS, 146, 217

Massey, P., \& Thompson, A. B. 1991, AJ, 101, 1408

Massey, P., Johnson, K. E., \& Degioia-Eastwood, K. 1995, ApJ, 454,151

McDowell, J. C. 1994, The Einstein Observatory Soft X-ray Source List, SAO HEAO CD-ROM Series

Miralles, M. P., Rodríguez, L. F., \& Scalise, E. 1994, ApJS, 92, 173

Morris, P. W., Eenens, P. R. J., Hanson, M. M., Conti, P. S., \& Blum, R. D. 1996, ApJ, 470, 597

Odenwald, S. F. 1989, AJ, 97, 801

Odenwald, S. F., \& Schwartz, P. R. 1993, ApJ, 405, 706

Oliva, E., \& Origlia, L. 1992, A\&A, 254, 466

Palla, F., Brand, J., Cesaroni, R., Comoretto, G., \& Felli, M. 1991, A\&A, 246, 249

Parthasarathy, M., \& Jain, S. K. 1995, A\&AS, 111, 407

Pigulski, A., \& Kolaczkowski, Z. 1998, MNRAS, 298, 753

Reddish, V. C., Lawrence, L. C., \& Pratt, N. M. 1966, Pub. Royal Obs. Edinburgh, 5, 111

Rieke, G. H., \& Lebofsky, M. J. 1985, ApJ, 288, 618

del Río, E., von Ballmoos, P., Bennett, K., et al. 1996, A\&A, 315,237

Schulte, D. H. 1958, ApJ, 128, 41

Thé, P. S., de Winter, D., \& Pérez, M. R. 1994, A\&AS, 104, 315

Tokunaga, A. T. 2000, in Allen's Astrophysical Quantities, ed. A. N. Cox, AIP Press

Torres-Dodgen, A. V., Tapia, M., \& Carroll, M. 1991, MNRAS, 249, 1

Vaiana, G. S., Cassinelli, J. P., Fabbiano, G., et al. 1981, ApJ, 245, 163

Walborn, N. R., \& Howarth, I. D. 2000, PASP, 112, 1446

Waldron, W. L., Corcoran, M. F., Drake, S. A., \& Smale, A. P. 1998, ApJS, 118, 217

Wendker, H. J., Higgs, L. A., \& Landecker, T. L. 1991, A\&A, 241,551 